\title{
Joint effects of magnitude of immediate reward and magnitude of delayed reward on acquisition and extinction'
}

\author{
E. J. CAPALDI, R. C. GODBOUT, and BARBARA DOWNS, \\ University of Texas, Austin. Texas 78712
}

An irregular schedule of partial delay of reward was employed. Magnitude of reward was either large or small on delay-of-reward trials and on immediately-rewarded trials. Speed in acquisition was independent of magnitude of delayed reward, but was greater the greater the magnitude of immediate reward. Resistance to extinction increased as magnitude of immediate reward increased and decreased as magnitude of delayed reward increased.

Partial reward and partial delay of reward are similar in that both kinds of schedules contain immediately rewarded trials. They differ in containing either nonrewarded trials (partial reward) or delay of reward trials (partial delay). In partial reward, magnitude of reward can be varied only on immediately rewarded trials. However, in partial delay, reward magnitude may be varied on both immediate trials and delay trials. The present experimental design, a 2 by 2 factorial, was such that reward magnitude was either large or small on immediate trials and on delay trials.

Under irregular schedules of partial reward, resistance to extinction was shown to increase as magnitude of immediate reward increased (Hulse, 1958; Wagner, 1961). Magnitude of immediate reward should affect resistance to extinction in the same way in a partial delay schedule (Amsel, 1958; Capaldi, 1967).

It has been assumed (Capaldi, 1967) that as magnitude of delayed reward decreases, it becomes increasingly dissimilar to immediate reward and increasingly similar to nonreward. This suggests that in a partial delay schedule, resistance to extinction should increase as magnitude of delayed reward decreases.

\section{METHOD}

The Ss were 44 male Holtzman rats about 90 days of age. The apparatus was a gray runway, $82 \mathrm{in}$. long, $4 \mathrm{in}$. wide with 9 in. sides covered with hardware cloth. The initial portion of the alley was a 10-in. floor treadle which, when depressed by the weight of the rat, whose front paws were placed at its forward edge, started the start clock $(.01 \mathrm{sec})$. When $S$ moved forward 4 in.. he broke a photobeam which stopped the start section clock and started the run section clock. The run section clock was stopped and the goal section clock started when $S$ broke a second photobeam, 52 in. from the first one. Breaking the third photobeam, which was located 2 in. from a $2 \times 4 \frac{1}{2} \times 1 \frac{1}{2} \mathrm{in}$. food cup, stopped the goal section clock and resulted in food pellets being delivered either immediately or $30 \mathrm{sec}$ later. A brass guillotine door was lowered behind $\mathrm{S}$ to confine it within the goalbox.

Following five days on a $12-\mathrm{g}$ deprivation cycle, Ss were given 10 days of pretraining consisting of handling. exploration of the alley, and goalbox placements on which $\mathrm{S}$ was allowed to eat six $.045 \mathrm{~g}$ Noyes pellets.

Experimental training consisted of 24 days of acquisition followed by 28 days of extinction, all at one trial each day. All Ss were given $50 \%$ partial delay of reward in acquisition under an irregular schedule such that no more than two delay-of-reward trials or two immediately rewarded trials occurred in succession. The delay was always $30 \mathrm{sec}$. There were two levels of reward magnitude on immediately rewarded trials ( 2 vs $24 \quad .045-\mathrm{g}$ pellets) and two levels of reward magnitude on delay of reward trials (2 vs 24 pellets), generating a 2 by 2 factorial design. The four groups of $11 \mathrm{Ss}$ each were designated $D_{2} I_{2}$ (two pellets reward on both delayed and immediate trials), $\mathrm{D}_{2} \mathrm{I}_{24}, \mathrm{D}_{24} \mathrm{I}_{2}$ and $\mathrm{D}_{24} \mathrm{I}_{24}$. The amount eaten in the runway was subtracted from the $12 \mathrm{~g}$ daily ration, which was always given $15 \mathrm{~min}$ following the daily training trial. The $S$ was allowed $60 \mathrm{sec}$ to traverse each section of the runway before being forced into the next section. From the goal section $S$ was forced into the goalbox. The $S$ was removed from the goalbox immediately after having eaten in acquisition and following a $30 \mathrm{sec}$ confinement in extinction.

\section{RESULTS}

\section{Acquisition}

All times for the start, run, and goal measures were converted to speeds ( $1 /$ time), and a total speed measure was obtained by reciprocating the sum of a S's start, run, and goal times on a particular trial. All statistical analyses are based on $1 / 40 \mathrm{df}$. An analysis based on the last three trials of acquisition and the first trial of extinction was performed. It indicated that terminal acquisition speeds were not affected either by magnitude of delayed reward or the interaction of immediate reward and delayed reward. Terminal acquisition speed was influenced, however, by magnitude of immediate reward, being greater in all alley sections the greater the magnitude of immediate reward (start $F=30.21, p<.001$, run $F=28.69, p<.001$, goal $F=7.63, p<.01)$. In view of the acquisition differences the extinction speeds were converted to rate measures as recommended by Anderson (1963). Acquisition asymptote for an $\mathrm{S}$ was its mean speed on the last three trials of acquisition and the first trial of extinction. The extinction asymptote was common for all Ss, being the reciprocal of the "forcing time" ( $60 \mathrm{sec}$ in start, run and goal. $180 \mathrm{sec}$ for total).

Extinction

Figure 1 shows rate measures based on the total speed measure in blocks of two trials. Statistical analyses support

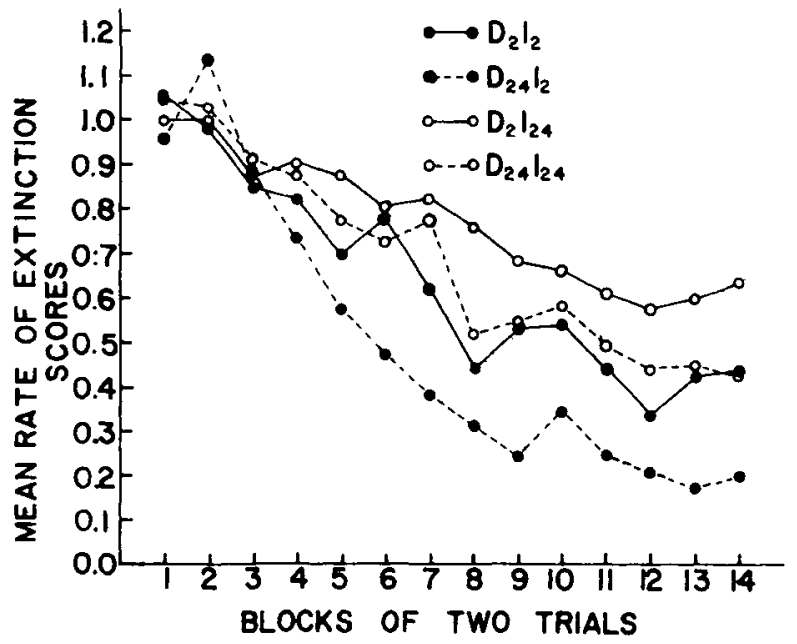

Fig. 1. Mean rate of extinction scores for the total speed measure for each of the four experimental groups in blocks of two trials. 
what can be seen in Fig. 1, that resistance to extinction (a) increased as magnitude of immediate reward increased $(F=28.05, p<.001)$, (b) decreased as magnitude of delayed reward increased $(F=14.48, p<.001)$ and $(c)$ was independent of the $D$ by $I$ interaction $(F<1)$.

The section results (not shown) indicated that magnitude of delayed reward and immediate reward affected extinction in exactly opposite ways over alley sections. The influence of magnitude of immediate reward declined from start to goal (start $F=8.83, p<.01 ;$ run $F=8.87, p<.01$; goal $F=1.51$, N.S.). The influence of magnitude of delayed reward, however, increased from start to goal (start $F=1.14$, N.S.; run $F=19.96$, $\mathrm{p}<.001$, goal $\mathrm{F}=22.59, \mathrm{p}<.001)$.

\section{DISCUSSION}

Resistance to extinction increased as magnitude of immediate reward increased and decreased as magnitude of delayed reward increased. In a partial delay schedule, then, magnitude of immediate reward and magnitude of delayed reward produced opposite effects on extinction. The present results are deducible from a sequential hypothesis. The basis of this deduction has been considered in detail elsewhere (Capaldi, 1967).

Increased magnitude of immediate reward produced the same effect in an irregular partial delay schedule as it already has been shown to produce in an irregular partial reward schedule, i.e., it increased resistance to extinction (Hulse, 1958; Wagner, 1961). Leonard (in press) has shown, however, that under other than irregular schedules of partial reward, increased magnitude of reward may produce decreased resistance to extinction. A subsequent investigation (Capaldi \& Lynch, 1968) confirmed that the effects on extinction of magnitude of partial reward depend upon schedule of partial reward. Possibly, then, the present findings are as peculiar to irregular schedules of partial delay as the Hulse-Wagner type of finding is peculiar to irregular schedules of partial reward. Two somewhat different explanations of the relationship between magnitude of reward and schedule of reward have been considered elsewhere (Capaldi, 1967; Leonard, in press).

Turning to the frustration hypothesis, it would most certainly deal with magnitude of immediate reward in a partial delay schedule as it has already dealt with magnitude of reward in a partial reward schedule (Amsel, 1962; Wagner, 1961). Regarding delayed reward, it would be expected that $r_{g}$ would increase as magnitude of delayed reward increased. In isolation this suggests that frustration and thus resistance to extinction should increase as magnitude of delayed reward increases. However, other considerations within the frustration framework, already developed by others, suggest a conclusion in line with the present results.

It is suggested that frustration can be related in the partial delay situation to the downward shift in $r_{g}$, an hypothesis originally entertained by Ludvigson \& Gay (1967) in the differential conditioning situation. The beginning of the delay interval (as $\mathrm{S}$ enters the goalbox) would be seen as the negative stimulus $(\mathrm{S}-)$. According to this view, $S$ traverses the alley under a relatively high $\mathrm{r}_{\mathrm{g}}$ level, which is determined by the reward magnitudes on both immediate and delay trials. On delay or $S$ - trials, the $r_{g}$ level drops from the alley level $(A)$ to the new level (B) which is determined by two aspects of delay, the delay interval itself and the reward magnitude following the termination of the delay interval. The downward shift in $r_{g}$ in the presence of $\mathrm{S}$ - (and hence frustration) could be assumed to be greater (a) the longer the delay interval and (b) the less the reward magnitude received at the termination of the delay interval. This analysis suggests, among other things, that resistance to extinction should increase as magnitude of delayed reward decreases.

\section{REFERENCES}

AMSEL, A. The role of frustrative nonreward in noncontinuous reward situations. Psychological Bulletin, 1958, 55, 102-119.

AMSEL, A. Frustrative nonreward in partial reinforcement and discrimination learning: Some recent history and a theoretical extension. Psychological Review, 1962, 69, 306-328.

ANDERSON, N. H. Comparison of different populations: Resistance to extinction and transfer. Psychological Review, 1963, 70, 162-179.

CAPALDI, E. J. A sequential hypothesis of instrumental learning. In K. W. Spence and J. T. Spence (Eds.), The psychology of learning and motivation: Advances in research and theory. Vol. 1. New York: Academic Press, Inc., 1967. Pp. 67-156.

CAPALDI, E. J., \& LYNCH, A. D. Magnitude of partial reward and resistance to extinction: Effect of N-R transitions. Journal of Comparative \& Physiological Psychology, 1968, 65, 179-181.

HULSE, S. H. Amount and percentage of reinforcement and duration of goal confinement in conditioning and extinction. Journal of Experimental Psychology, 1958, 56, 48-57.

LEONARD, D. W. Amount and sequence of reward in partial and continuous reinforcement. Journal of Comparative \& Physiological Psychology, in press.

LUDVIGSON, H. W., \& GAY, R. A. An investigation of conditions determining contrast effects in differential reward conditioning. Journal of Experimental Psychology, 1967, 75, 37-42.

WAGNER, A. R. Effects of amount and percentage of reinforcement and number of acquisition trials on conditioning and extinction. Journal of Experimental Psychology, 1961, 62, 234-242. NOTE

1. This research was supported in part by National Institute of Child Health and Human Development Research Grant HD00949-06 to the first author and by Public Health Service Fellowship 2-FI-MH-32, 183-03 (PS) from the National Institute of Mental Health to the second author. 